\title{
Supermassive binary black holes in blazars
}

\author{
Frank M. Rieger
}

Department of Mathematical Physics, University College Dublin, Belfield, Dublin 4, Ireland

\begin{abstract}
Low-frequency $\left(f \leqslant 10^{-6} \mathrm{~Hz}\right)$ quasi-periodic variability observed from radioloud, jet-emitting Active Galactic Nuclei (AGNs) can provide substantial inductive support for the presence of close $(d \lesssim 0.1 \mathrm{pc})$ supermassive binary black holes in their centers. Such periodic variability may arise, for example, due to differential Doppler boosting along helical jet paths driven by the orbital motion or jet precession. If the jet path is non-ballistic, travel time effects can lead to a strong reduction of the observable period by up to a factor of $\gamma_{b}^{-2}$. Here we suggest a binary model where the optical periodicity with timescale of several years is related to accretion disk interactions, radio periodicity to Newtonian jet precession and periodicities in the high energy bands to the orbital motion of the jet. We analyze the explanatory potential of such a framework and comment on the possible origin of periodicities in AO 0235+16.
\end{abstract}

Keywords. black hole physics - galaxies: active - galaxies: jets.

Supermassive binary black holes (SBBHs) are generally expected to form during the cosmological evolution of galaxies. In hierarchical clustering scenarios, for example, giant elliptical galaxies such as the host galaxies of radioloud AGNs are thought to be the product of mergers between spiral galaxies each containing its own black hole $(\mathrm{BH})$. Interacting galaxies are indeed observationally well-known and give strong reasons for such an evolutionary picture. In particular, direct observational support has been recently provided by the Chandra discovery of two wide activity centers (separation $d \sim 1 \mathrm{kpc}$ ) in the merging galaxy NGC 6240 (Komossa et al. 2003). While the existence of wide SBBHs seems thus well grounded, the existence of close $(d \sim 0.003-0.1 \mathrm{pc})$ SBBHs appears much more ambiguous. Dynamical friction and slingshot interactions with stars normally ensure that the binary BHs quickly get closer (e.g., Begelman et al. 1980). However, due to a 'loss cone depletion' it is still an open question today whether a substantial fraction of SBBHs can really coalesce within a Hubble time.

The presence of close SBBHs has been repeatedly invoked as plausible source for a number of observational findings in blazars (e.g., misalignment and precession of jets, apparent helical trajectories of knots or periodic variability; cf. Rieger 2005 for a review). Interactions of the companion with the accretion disk around the primary, for example, can provide a natural explanation for the longterm optical periodicity with periods $P_{\mathrm{obs}}^{\mathrm{opt}}$ of several years as observed in a number of blazars. It seems likely that the combined effects of dynamical friction, accretion disk interactions and gravitational radiation produce orbits that are nearly circular. Assuming that the observed periodicity is caused by the secondary $\mathrm{BH}$ crossing the disk around the primary $\mathrm{BH}$ on a slightly non-coplanar orbit, we can derive an upper limit for the real Keplerian orbital period $P_{k} \simeq 2 /(1+z) P_{\mathrm{obs}}^{\mathrm{opt}}$. The observations of helical jet paths in many blazar sources suggest that periodic variability (especially in the energy range dominated by the jet, e.g., radio, $\mathrm{X}$ - and $\gamma$-ray) can also arise due to differential Doppler boosting for a periodically changing viewing angle. For non-ballistic motion, travel time effects will lead to a reduction of the observable period $P_{\text {obs }}$ with respect to the real driving period $P$ such that $P_{\text {obs }} \simeq(1+z) P / \gamma_{b}^{2}$, where $\gamma_{b} \sim(5-15)$ is the bulk flow Lorentz factor (Rieger 2004). The orbital motion of the jetemitting $\mathrm{BH}$ and (Newtonian) precession of the jet generally represent the most obvious 
Table 1. Properties of a sample of blazar SBBH candidates, cf. Rieger (2005) for details.

\begin{tabular}{|c|c|c|c|c|c|}
\hline name & redshift $z$ & periods $P_{\mathrm{obs}}$ & $(m+M) / 10^{8} M_{\odot}$ & $P_{k}[\mathrm{yr}]$ & $d / 10^{16} \mathrm{~cm}$ \\
\hline Mkn 501 & 0.034 & $\begin{array}{l}23.6 \mathrm{~d} \text { (X-ray) } \\
\sim 23 \mathrm{~d}(\mathrm{TeV})\end{array}$ & $(2-7)$ & $(6-14)$ & $(2.5-6)$ \\
\hline BL Lac & 0.069 & $\begin{array}{l}13.97 \text { yr (optical) } \\
\sim 4 \text { yr (radio) }\end{array}$ & $(2-4)$ & $(13-26.1)$ & $(4.8-9.7)$ \\
\hline $3 \mathrm{C} 273$ & 0.158 & $\begin{array}{l}13.65 \text { yr (optical) } \\
8.55 \text { yr (radio) }\end{array}$ & $(6-10)$ & $(11.8-23.5)$ & $(6.5-12)$ \\
\hline OJ 287 & 0.306 & $\begin{array}{l}11.86 \text { yr (optical) } \\
\sim 12 \text { yr (infrared) } \\
\sim 1.66 \text { yr (radio) }\end{array}$ & 6.2 & $(9.1-18.2)$ & $(5.5-8.8)$ \\
\hline $0235+16$ & 0.940 & $\begin{array}{l}2.95 \mathrm{yr} \text { (optical)? } \\
5.7 \mathrm{yr} \text { (radio) }\end{array}$ & $\geqslant 1$ & $(1.5-3.1)$ & $\geqslant 0.95$ \\
\hline
\end{tabular}

driving sources for helical jet paths. Whereas the high energy emission is usually produced on small jet scales and thus likely to be modulated by the orbital motion of the SBBHs so that $P_{\mathrm{obs}} \sim 30\left(P_{\mathrm{obs}}^{\mathrm{opt}} / 10 \mathrm{yr}\right)\left(15 / \gamma_{b}\right)^{2} \mathrm{~d}$, the main part of the radio jet emission usually originates from larger scales where the jet motion is likely to be dominated by Newtonian precession with a period which is (at least) an order of magnitude higher than $P_{k}$, i.e., $P=P_{p} \gtrsim 10 P_{k}$ (Rieger 2004), so that one finds $P_{\text {obs }}^{\text {radio }} \gtrsim 20 P_{\text {obs }}^{\text {opt }} / \gamma_{b}^{2}$. Hence, if $P_{p}$ is rather small (say $P_{p} \sim 10 P_{k}$ ), moderate bulk Lorentz factors can be sufficient to account for $P_{\mathrm{obs}}^{\text {radio }}<P_{\mathrm{obs}}^{\mathrm{opt}}$. Table 1 shows properties of a sample of blazar SBBH candidates, where the observed periodicities have been used to estimate the last two columns.

The results above may be particularly relevant for an appropriate understanding of the nature of the BL Lac AO $0235+16$ (cf. Table 1), where two different SBBH models have been proposed recently (cf. also Rieger 2005): (i) In Romero et al. (2003) the observed optical periodicity has been related to accretion disk interactions (implying $\left.P_{k} \simeq 2 \times 2.95 /[1+z] \sim 3 \mathrm{yr}\right)$ and the radio one to Newtonian jet precession. (ii) In Ostorero et al. (2004) the radio and optical periodicity has been related to a helically bent inhomogeneous jet, driven by the orbital motion (using the radio period as direct tracer). Scenario (i) requires that the jet fluid motion is non-ballistic with $\gamma_{b}>3$ as otherwise $P_{p}$ would be too short. Moreover, the orbital motion should lead to some quasi-periodic modulation in the high energy bands, with observable periods ranging from $\sim 7$ months (for $\gamma_{b} \sim 3$ ) to $\sim 20$ days (for $\gamma_{b} \sim 10$ ). Scenario (ii), on the other hand, seems less plausible if $P_{\mathrm{obs}}^{\mathrm{opt}} \neq P_{\mathrm{obs}}^{\text {radio }}$ (cf. Table 1), as is indeed confirmed by further studies. It is likely, however, that the real situation is much more complex, e.g., the radio jet flow may repeatedly approach the line-of-sight, resulting in a maximization of beaming effects ("radio knots") and an apparent short Keplerian period, e.g., $P_{\text {obs }}^{\text {radio }} \simeq 5.7$ yr may in reality imply $P_{k} \simeq 26 \mathrm{yr}$ (for $\gamma_{b}=3$ ), and the radio lightcurves may have pronounced peaks separated by $(1+z) P_{k}$, with intermediate peaks occurring every $5.7 \mathrm{yr}$.

\section{References}

Begelman, M. C., Blandford, R. D. \& Rees, M. J. 1980, Nature 287, 307

Komossa, S., Burwitz, V., Hasinger, G. et al. 2003, ApJ (Letters) 582, L15

Ostorero, L., Villata, M. \& Raiteri, C.M. 2004, A\&A 419, 913

Rieger, F.M. 2004, ApJ (Letters) 615, L5

Rieger, F.M. 2005, Proc. 22nd Texas Symposium on Relativistic Astrophysics, Stanford, eds. P. Chen et al. (eConf:C041213), 1601

Romero, G.E., Fan, J. \& Nuza, S. E. 2003, ChJAA 3, 513 